\title{
TOWARDS OPERATIONALIZING DRIVER DISTRACTION
}

\author{
James P Foley ${ }^{1}$, Richard Young ${ }^{2}$, Linda Angell ${ }^{3}$ \& Joshua E Domeyer ${ }^{1}$ \\ ${ }^{1}$ Toyota Collaborative Safety Research Center, Ann Arbor, MI \\ ${ }^{2}$ Wayne State School of Medicine, Detroit, MI \\ ${ }^{3}$ Virginia Tech Transportation Institute, Blacksburg, VA \\ Email: James.Foley@tema.toyota.com
}

\begin{abstract}
Summary: Driver distraction has been the subject of much research interest and scientific inquiry. Operationalizing driver distraction is a complex task-one that is necessary for advancing both science and public policy in this domain. While many operational definitions can be gathered from the literature, gaps are common. In order to fill such gaps, 21 experts reviewed 55 driver distraction definitions in the literature. Aided by the results of a pre-workshop questionnaire the experts narrowed these definitions. The Regan et al. (2011) definition of driver distraction was agreed to at a workshop. Subsidiary terms related to this definition were defined to improve clarity and applicability of the definition. It is hoped that a consistent and agreed definition of driver distraction and its associated terms will advance scientific progress in understanding and measuring driver distraction.
\end{abstract}

\section{INTRODUCTION}

Mitigating driver distraction is a focus of the US Department of Transportation (see www.distraction.gov) and the National Highway Traffic Safety Administration (NHTSA, 2012), automobile manufacturers (The Alliance, 2006), other vehicle safety-concerned organizations, and the general public. Driver distraction has also been (and still is) the subject of much research interest and scientific inquiry (e.g., Lee, Young \& Regan, 2009; Rupp, 2010). However, there is not a consistent or agreed definition of driver distraction. However, this is needed for scientific advancement in understanding driver distraction and driving assessment in general. Existing definitions are contradictory or incomplete, and/or use terms such as "attention” or "workload" without a scientific definition:

"Historically, driving performance measures have been undefined or vaguely defined, and if defined, inconsistently used. Consequently, driving studies are difficult to compare and challenging to replicate, undermining the professional credibility of researchers and the field of automotive human factors.” (Savino, 2009)

There are many benefits of having an agreed scientific definition:

"An agreed definition would help to focus clarity within future research, making crossstudy comparisons easier. Such a definition may be particularly useful for accident researchers seeking to categorize cases, allowing for comparisons between statistics from different areas, regions, countries etc.” (Pettitt, 2005, p. 2) 
"The naming/identification of the problem is important because of its implications for what one thinks the problem is and which performance measures should be collected.” (Green, 2010)

Toyota’s Collaborative Safety Research Center (CSRC) convened a workshop to improve definitions of driver distraction in the scientific community by obtaining input from selected invitees with expertise in driver distraction, i.e., actively engaged in relevant research for at least 5 years, to generate common terminology for all researchers in the field of driver distraction. This paper describes the methods, key outcomes, and the consensus on the definition of driver distraction that emerged from the workshop.

\section{METHOD}

Five key efforts preceded the workshop:

1. Preparing a clear definition of the goals of the project,

2. Conducting a literature search for existing driver distraction definitions,

3. Constructing a questionnaire to identify the "best" definitions currenlty in the literature,

4. Distributing the questionnaire to invited participants, and

5. Analyzing the results of that questionnaire to identify initial points of consensus.

\section{Goals}

The goals of this undertaking were initially to reach consensus on 3 definitions:

1. Top-level definition of driver distraction,

2. Definition of visual-manual distraction,

3. Definition of auditory-vocal-cognitive distraction.

\section{Literature Search}

A comprehensive literature search of databases and key articles in English, Japanese, and French was conducted by the second author, aided by a librarian search for key words in those languages. A total of 55 published definitions of driver distraction were found. The list of definitons is available at www.toyota.com/csrc.

\section{Questionnaire Method}

From these 55 definitions, 27 questions that discriminated between them were created (available at www.toyota.com/csrc). The questions were grouped into general questions, scope questions, and terms to be included in the definition or not. For example, the first question was: "Must driver distraction include a trigger?”

The term trigger was defined as "an object, person, task, activity, event, happening, movement, process, condition, situation, source, or agent that sets off a mechanism that causes, precipitates, or initiates a mental event, such as inducing the driver's shifting of attention away from the driving task, or cause other changes in mental processing.” About half the 55 definitions of 
distraction invoked or required a trigger, and half did not. Based on the questionnaire responses to just this one question, half the definitions were eliminated.

A questionnaire was sent to all invited attendees prior to the workshop. Sixteen experts completed the questionnaire. All of the definitions were filtered based on the experts' responses to the 27 questions. Preliminary analysis found that no one definition remained that met all the experts' answers. Therefore, the 5 point rating system shown in Table 1 was used. Adding the scores for the 55 definitions across all 27 questions provided a ranking of definitions prior to the workshop. The top two canidates were then selected for discussion at the workshop. It is important to note that the results of the questionnaire served solely as a starting point to facilitate the workshop discussions and make optimal use of workshop time. If an attribute was selected $a$ priori by the experts, it was not necessarily required to be part of the final definition, which was acheived during the workshop.

Table 1. Scoring system for responses

\begin{tabular}{cl}
\hline Value & \multicolumn{1}{c}{ Criterion } \\
\hline+2 & Explicitly fit with 2/3 majority \\
+1 & Implicitly fit with 2/3 majority \\
0 & Not applicable, ambiguous, or blank \\
-1 & Implicitly disagreed with 2/3 majority \\
-2 & Explicitly disagreed with 2/3 majority \\
\hline
\end{tabular}

\section{Workshop Method}

Following the presentation of the questionnaire results and top candidate definitions, participants worked to reach consensus on a single definition of driver distraction. Use of the questionnaire results quickly focused the discussion on those definitions that were most relevant and aided the group in agreeing to the top-level definition. Considerable discussion then took place to define the subsidiary terms. Two smaller break-out groups were formed to define the classical distinction of driver distraction into visual-manual and auditory-vocal-cognitive distraction (Goals 2 and 3). The group then re-assembled to finalize the subsidiary definitions from the smaller groups in the context of the top-level definition.

\section{RESULTS}

\section{Questionnaire Results}

Table 2 provides the percent of expert agreement on terms that should be in the scope. Only drowsiness failed to be included in the scope.

After all of the questions were tabulate the deinitions were scored and placed in rank order.(see www.toyota.com/csrc for scoring). The two definitions with the highest score were selected for discussion. These were those from Regan et al. (2011) and Hurts, Angell, \& Perez (2011). These were also the two most recent definitions in the literature (as of the date of the workshop). 
Table 2. Scope of “distraction”

\begin{tabular}{rlr}
\hline 1. & Drowsiness & $21 \%$ \\
2. & Daydreaming & $79 \%$ \\
3. & Look-but-did-not-see & $79 \%$ \\
4. & Thoughts & $86 \%$ \\
5. & Passenger conversation & $86 \%$ \\
6. & Mental activity & $93 \%$ \\
7. & Hand-held cellular conversation & $100 \%$ \\
8. & Hands-free cellular conversation & $100 \%$ \\
9. & Manual or motor & $100 \%$ \\
10. & Auditory & $100 \%$ \\
11. & External distraction & $100 \%$ \\
12. & Driving-related tasks & $100 \%$ \\
\hline
\end{tabular}

\section{Goal 1: Top-level Definition of Driver Distraction}

After considerable discussion all experts agreed that Regan et al. (2011, p. 1776) was the best definition:

Driver distraction is the diversion of attention away from activities critical for safe driving toward a competing activity, which may result in insufficient or no attention to activities critical for safe driving.

\section{Workshop Results}

The experts agreed to clarify that "distraction" excludes human conditions or states that impair or alter the ability of a driver to function as an information processor (e.g., alcohol or drug impairment, drowsy driving, etc.). It was also recognized that drowsiness (or other states) may interact with distraction to impact resource availability and overall level of attention. This toplevel definition included some subsidiary terms that needed clarification. For example, this agreed definition of driver distraction did not provide a scientific definition of attention.

\section{Goals 2 and 3: Visual-manual vs. Auditory-vocal-cognitive Subsidiary Definitions}

More specific definitions of subsidiary terms, including driver resources, allows for individual coding of resource demands that may give rise (individually or in various combinations) to competing activities that may interfere with safe driving. In order to accommodate this principle, a different approach was taken to diverge from the conventional visual-manual and auditoryvocal-cognitive framework. However, conventional definitions are still consistent with the new framework. This new approach allows for the examination of a broader set of types of distraction (that may arise from mixed-mode interfaces which may place demands on visual, manual, auditory, vocal and cognitive resources of the driver). Five key outputs from the two working groups are presented below. 
1. Attention consists of the functions which arise from the brain networks that regulate and are involved in orienting, executive, and alerting, see Posner and Fan (2008):

o Orienting attention is defined as the selection of information from sensory input.

o Executive attention is defined as resolving conflict among responses, as well as regulating or modulating other network functions.

o Alerting attention is defined as achieving and maintaining an alert state.

2. Safe driving is operating a motor vehicle in a reasonable and expected manner.

3. Competing activity is an activity or activities which place/s demands upon cognitive, auditory, vocal/verbal, visual, motoric, and other resources, separately or in any combination - demands that are the same as or similar to the resources demanded by safe driving (hence giving rise to resource-competition), and which occur concurrently with driving.

\section{Resources are:}

o Cognitive - The alerting, executive, and orienting attentional networks singly or in combination, as well as the memory and representational systems (e.g., working and longterm) from which information may be retrieved and in which it may be held and operated upon.

o Auditory - The sensory organs and associated neurological structures, pathways, and processes by which hearing and perceiving sound occurs.

o Vocal/verbal - The structures, pathways, and processes associated with speaking, verbalizing, or making utterances covertly or overtly.

o Visual - The visual sensory organs and associated neurological structures, pathways, and processes.

o Motoric - The motor/biomechanical system and associated structures of movement within the body.

o Other - While not typically including in the discussion of resources in the context of driving, activation of these "other" resources may also impact attention and result in distraction:

5. Insufficient or no attention to activities critical for safe driving includes situations in which a driver is delayed in the recognition of information needed to safely accomplish the driving task.

\section{Applying These Definitions to Types of Distraction}

Using the definitions above, types of distraction can either be defined individually or in terms of combinations (e.g. "visual-manual” or "auditory-vocal-cognitive”). A combination definition of distraction such as "visual-manual task loading” acknowledges that visual-manual distraction results from high (competing) demands on visual and motoric resources, and can be specifically defined as "any glance and/or physical manipulation that competes with activities necessary for safe driving.”

However, based on the workshop consensus, there can be two separate definitions -- one for "visual distraction" - and one for "manual distraction." The purpose is to allow each element to be specifically coded from data, in order to more fully capture each type of competing resource 
demand associated with a potentially distracting task. This may extend the ability of researchers to capture and code complex tasks, which impose demands of several types on driver resources, in varied combinations. Table 3 shows an example of what subsidiary definitions might look like.

Table 3. Examples of subsidiary definitions

\begin{tabular}{ll}
\hline Specific Type & \multicolumn{1}{c}{ Definition } \\
\hline Visual Distraction & "Visual Distraction is any glance that competes with activities necessary for safe driving." \\
Manual Distraction & $\begin{array}{l}\text { "Manual Distraction is any physical manipulation that competes with activities necessary for } \\
\text { safe driving." } \\
\text { "Auditory Distraction is any period of aural stimulation that competes with activities necessary } \\
\text { for safe driving." } \\
\text { "Vocal Distraction is any vocal utterance (or covert sub-vocal utterance) that competes with } \\
\text { Vocal Distraction }\end{array}$ \\
Cognitive Distraction & $\begin{array}{l}\text { "Cognitive Distraction is any epoch of cognitive loading that competes with activities necessary } \\
\text { for safe driving." }\end{array}$ \\
\hline
\end{tabular}

\section{DISCUSSSION}

A unique feature of this driver distraction definition workshop was the extensive preparation to find the major areas of agreement prior to the workshop. Hence, a unanimous agreement was reached with mutual facilitation and positive contributions from all attendees.

Final agreement was achieved on the Regan et al. (2011) top-level definition of driver distraction. This definition was extended and enhanced by defining a number of subsidiary terms not included in the original definition. In addition, a new definition of competing activity in terms of a comprehensive list of resources was developed. Finally, a framework for defining distraction in terms of specific competing resource demands, laid the foundation for a new approach to coding and capturing the complexity of tasks that may lead to distraction. This approach represents a movement away from the often used visual-manual vs. cognitive (auditory/vocal) dichotomy, and is based on current neuroscience understanding of attention and distraction. Visual-manual vs. auditory-vocal definitions are useful; however, a refined understanding of attention, demand, resources and distraction is broader and provides a more neuro-biologically grounded understanding of resource involvement and interaction. In particular, defining cognitive demand for auditory, verbal-vocal tasks must not preclude cognitive demand being a component of visual-manual tasks. That is any activity a driver engages in has some cognitive component, even if it is quite small. Thus, the distinction between the auditory-vocal and visual-manual task definitions is more appropriately described by what resource loadings are present, their magnitude, and whether or not they cause conflict with resources required for safe driving.

\section{SUMMARY}

In summary the workshop procedure was highly successful and:

o Demonstrated that pre-planning and administering the questionnaire prior to the workshop provided a good foundation for discussions leading to rapid consensus.

o Achieved the goal of drafting common terminology for driver distraction research. 
o Clarified the Regan definition by defining related subsidiary terms.

o Established the benefits of moving beyond a dichotomous (visual-manual vs. cognitive) definition of tasks / resources, thus providing a more useful framework for identifying and coding all types of driver distraction.

o Succeeded in building agreement among a group of leading researchers in the distraction field.

A next step is to determine if these definitions can be successfully used to create a "third-level" of operational and observable definitions and determine if those definitions are useful for analyzing naturalistic driving data based on video and instrumented recordings.

\section{ACKNOWLEDGMENTS}

Funding was provided by the Toyota CSRC. The authors thank the following editing committee members: D. McGehee, B. Mehler, J. Owens, and B. Reimer. We also thank the other workshop attendees.

\section{REFERENCES}

Alliance. (2006). Alliance of Automobile Manufacturers Driver Focus-Telematics Working Group. Statement of Principles, Criteria and Verification Procedures on Driver-Interactions with Advanced In-Vehicle Information and Communication Systems, June 26, 2006 version. Wash., DC.

Green, P. A. (2010). Driver Distraction/Overload Research and Engineering: Problems and Solutions. SAE International Journal of Passenger Cars - Electronic and Electrical Systems, 3(2), 141-153.

Hurts, K., Angell, L. S., \& Perez, M. A. (2011). The Distracted Driver: Mechanisms, models and measurement Reviews of Human Factors and Ergonomics (Vol. 7, pp. 3-57). Santa Monica, CA: SAGE/Human Factors and Ergonomics Society.

Lee, J. D., Young, K. L., \& Regan, M. A. (2009). Defining driver distraction. In M. A. Regan, J. D. Lee \& K. L. Young (Eds.), Driver Distraction: Theory, Effects and Mitigation (pp. 31-40). Boca Raton, Florida USA: CRC Press.

NHTSA. (2012). Visual-Manual NHTSA Driver Distraction Guidelines for In-Vehicle Electronic Devices (Docket No. NHTSA-2010-0053). Washington, DC: National Highway Traffic Safety Administration. http://www.gpo.gov/fdsys/pkg/FR-2012-02-24/pdf/2012-4017.pdf.

Pettitt, M., Burnett, G., \& Stevens, A. (2005). Defining driver distraction. Paper presented at the 12th World Congress on Intelligent Transport Systems, San Francisco, CA, USA.

Posner, M. I., \& Fan, J. (2008). Attention as an organ system. In J. R. Pomerantz (Ed.), Topics in integrative neuroscience: From cells to cognition (pp. 31-61). Cambridge, UK: Cambridge University Press.

Regan, M. A., Hallett, C., \& Gordon, C. P. (2011). Driver distraction and driver inattention: Definition, relationship and taxonomy. Accident Analysis \& Prevention, 43(5), 1771-1781

Rupp, G. L. (Ed.). (2010). Performance Metrics for Assessing Driver Distraction: The Quest for Improved Road Safety. Warrendale, PA: SAE International. 